\title{
Optical Fibre Technology at STL
}

\section{An EPS Associate Member}

\section{Martin Chown and K. Ross Richmond, Harlow}

Standard Telecommunication Laboratories (STL), which is the research centre of STC, was set up in 1946 at Enfield and moved to Harlow (Essex) in 1959. There are currently over a thousand employees, a significant proportion of whom work on one or other of the many disciplines of optical fibre technology.

The principle that light would follow the curved jet of water issuing from a container was demonstrated in 1870 to members of the Royal Society. Bell envisaged a free-space, optical telephone. More recently, John Logie Baird filed patents covering the transmission of light in glass rods. But the practical implementation of glass fibres as transmission media did not become a realistic proposition until the late 1950s when they were used for the transmission of visible light for remote illumination, or in flexible viewing instruments for medical applications.

Optical communications only became the goal of telecommunication engineers with the invention of the laser in 1960. First experiments on a guiding medium included confocal lens systems and thermally controlled gas-filled pipes (producing a graded refractive index across a cross-section), the concept being that enormous information bandwidths would justify the high cost of such trunk links. Optical fibres, at this time, were seen as short distance light guides, as attenuations were of the order of hundreds of dB/km. In 1966 Kao and Hockham ${ }^{1}$ ) working at STL, analysed glass fibre as a single-mode long distance dielectric waveguide, and concluded that impurities should be reducible sufficiently to make it a suitable medium for optical transmission, in con-

Fig. 1 - Refractive index profile of an optical fibre preform.

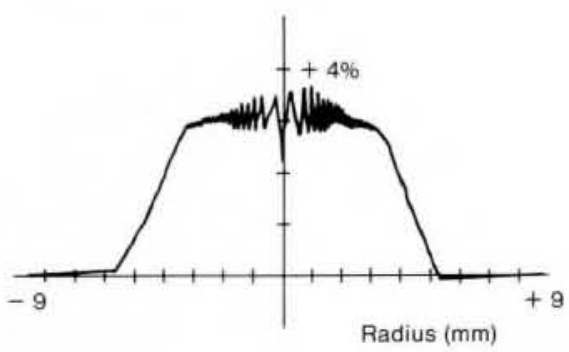

junction with semiconductor lasers which were also being developed at STL. The company accepted this judgment, and discontinued not only the confocal lens experiments, but even more boldly abandoned its strong position in circular copper trunk waveguide for microwaves, generally seen as the next generation for trunk lines at that time.

By 1969, STL had demonstrated a $100 \mathrm{Mbit} / \mathrm{s}$ repeater using cooled lasers and very short lengths of fibres and in 1971, in conjunction with the BBC's digital coding equipment, the first digital colour TV link. The 1970s saw development of the full range of relevant techno logies including low loss fibres, electrooptic and passive devices, and their exploitation in a very wide range of systems. The advantages to the user have turned out to be much more far-reaching than the length and bandwidth capability offer, for example immunity to electromagnetic interference has resulted in optical fibres being exploited in many more industrial, military and telecommunications applications than were originally envisaged.

\section{Fibre and Cable}

Long-haul optical communications using single-mode fibres require a fibre which has very low loss over the required wavelength range, compatibility with the cabling process, and well controlled chromatic dispersion. Core and cladding dimensions together with low eccentricity must be controlled within low tolerances during the manufacturing process. Moreover, for economy of manufacture and to minimise the need for in-line splicing, the longest possible lengths of fibre should be drawn from each preform.

A thin plastic coating is applied as part of the fibre pulling process, the primary objective being to maintain the pristine surface quality of the fibre as pulled, and so maintain strength. Such a coating must be free from any flaws (such as particles) and act as a chemical barrier against surface attack over the fibre life, be compatible with splicing and connecting techniques and also be compatible with the overall mechanical protection

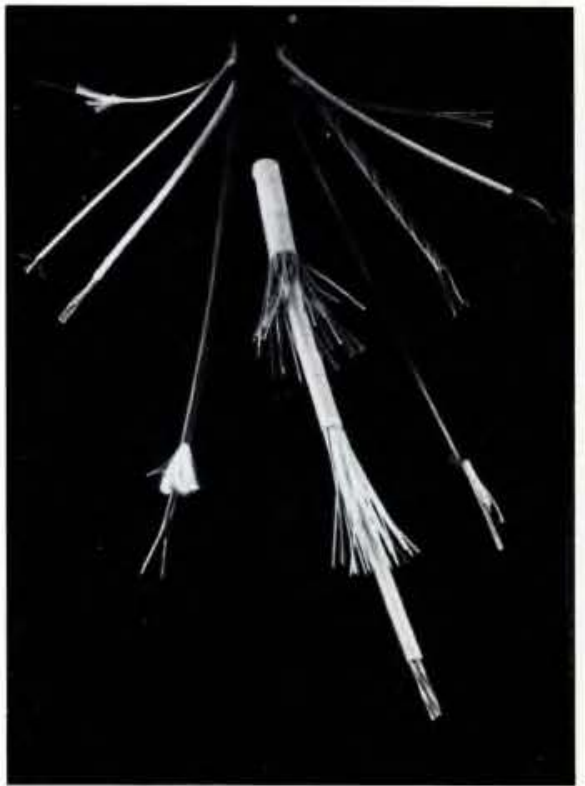

Fig. 2 - Optical cables are complex structures requiring refined production methods.

system - i.e. cabling - without inducing additional loss through microbending.

For the many shorter distance applications, different designs of fibre are appropriate. For example, in avionic or ship board data buses (where the attractions of optical fibres include immunity to electromagnetic effects) attenuation per kilometre is no longer the key issue, but efficiency of coupling through many connectors and splitters, typical of air craft wiring. For this reason, a larger core size and numerical aperture (related to acceptance angle of incoming rays) becomes more appropriate for this application. Fig. 1 shows the refractive index profile of a large core fibre. Further, nuclear hardness is important for this and other military applications.

Although optical fibres can be very strong for their dimensions, they need to be made into cables for nearly every ap plication. These must withstand the rigours of installation and must function reliably, often in extremely adverse en vironment. Cable types range from the heavy armoured cable designed to withstand trawler and anchor damage in continental shelf undersea applications, to very thin flexible single fibre types with extremely high strength to weight ratios capable of working at extremes of high temperature and pressure. An indication of the range of products is given in Fig. 2 and each application has its special challenges which could include incorporation alongside or within high voltage electrical conductors.

In addition, the cable has to be design ed with particular reference to connec- 
ting, repair, splicing and termination requirements, necessitating close collaboration with specialists in these fields and with the system designer.

The importance of characterising and measuring fibres and cables has long been recognised at STL, and an extensive measurement facility has been built up over 20 years in parallel with (and even predating) the development of fibres. These measurements cover the preform as well as pulled fibre stages. In addition to spectral attenuation (see Fig. 3 for a typical single-mode fibre loss spectrum), dispersion, spot-size, refractive-index profile and other tests are now standard.

Often for diagnosis, knowledge of the changes over the full spectrum from 0.7 to $2.0 \mu \mathrm{m}$ will be required and optical reflectometry may be essential to determine the character of such changes. Also, the determination of changes in the optical length gives information that is important. Such measurements are made by launching modulated laser light into a fibre and determining any changes in the phase of the modulation either at the far end or, after reflection, at the launch end. If for example, a signal of intensity $P \cos \omega t$ is launched into the fibre, a reflected signal $\alpha P \cos (\omega t-\phi)$ will be received where $\alpha$ represents attenuation and $\phi$ the delay. The two signals can be detected simultaneously and electronic phase comparison used to determine $\phi$, from which the length can be calculated for a known optical velocity. The actual method is slightly more refined than this, and results in detection of very small length changes between measurments at quite separate times. The ability to make such measurements single-ended has enabled changes in strain to be monitored at every stage in the cabling process and during all tests, giving vital information to the cabling engineer.

\section{Active Optical Components}

STL was one of the first laboratories to develop semiconductor lasers, with GaAs lasers operating in 1963, emitting at around $900 \mathrm{~nm}$ wavelength. At room temperature, these devices could operate at very low duty cycles, but when continuous operation became possible in 1970, it was immediately shown that they were capable of being modulated at $1 \mathrm{Gbit} / \mathrm{s}$ lon-off keying with $1 \mathrm{~ns}$ pulse period) by controlling the applied current. At that time, device life was very short indeed, but ensuring that the critical active layers were well embedded within the device, and paying attention to the light-emitting surfaces gave great

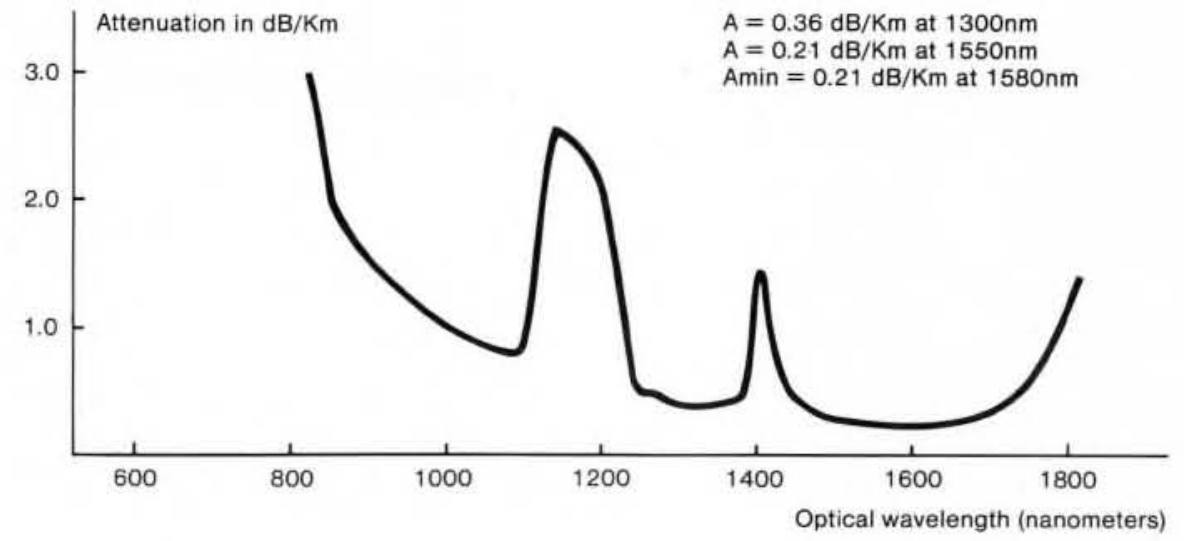

Fig. 3 - Typical spectral attenuation curve of an optical fibre.

\section{A basic source of numerical data and functional relationships in science and technology}

\section{Landolt-Börnstein LB \\ New Series}

Landolt-Börnstein is the only fully systematic and comprehensive collection of critically assessed DATA from all fields of physics, physical chemistry, geophysics, astronomy, material technology and engineering.

The New Series provide exhaustive data reviews on selected topics and are designed to meet the needs of today's specialist requiring well coordinated, concise and up-to-the-minute information in his field. Most of the New Series volumes cover areas which are at present developing rapidly and in which new findings are reported continuously.

The New Series are divided into six main groups, each of which is further divided into a series of volumes on particular topics. The number of volumes in each group is not fixed and is expanded to include new spheres of current scientific interest and/or revised appraisals and extensions of existing data as the need arises. At present 92 volumes of the New Series are available.

The 6 main groups cover:

\section{Nuclear and Particle Physics}

Energy levels, radii, decay, reactions of nuclei. Angular correlations. Properties, production and scattering of elementary particles, etc. (up to now: 13 volumes).

\section{Atomic and Molecular Physics}

Structure and molecular constants of free molecules, magnetic properties of free radicals and of coordination and organo-metallic transition metal compounds, luminescence, molecular acoustics, etc. (up to now: 24 volumes).

\section{Crystallography and Solid State Physics}

Crystal structure data of elements, intermetallic, inorganic and organic compounds. Linear and nonlinear elastic, dielectric, optic and coupled properties. Ferro- and antiferroelectric substances. Magnetic oxides and related compounds. Electron and phonon states of metals, etc. (up to now: 42 volumes).

\section{Macroscopic and Technical Properties}

Thermodynamic properties of liquid systems. High pressure properties of matter (up to now: 5 volumes).

V Geophysics and Space Research

(up to now: 4 volumes)

VI Astronomy and Astrophysics

(up to now: 4 volumes).

The following publications are available free of charge:

Brochure "What is Landolt-Börnstein?" - an attractively presented, informative booklet.

Landolt-Börnstein Reference Chart Poster.

Landolt-Börnstein Outline.

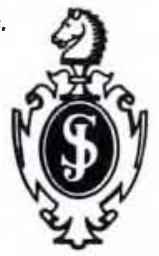

Springer-Verlag Berlin Heidelberg New York Tokyo

4005 - Marketing, Heidelberger Platz 3 D-1000 Berlin 33, Germany 
improvements. Detailed attention to understanding the physics of failure led to devices of commercial reliability, even for the most demanding undersea telephone cable applications. This was helped by ever reducing drive current requirements as new structures were developed ${ }^{2}$ ) with a minimum to date of $4.6 \mathrm{~mA}$ at more than $80 \%$ quantum efficiency.

The devices currently being installed in long-haul systems operate at 1300 $\mathrm{nm}$. The next generation, now well established in laboratories, operate at around $1550 \mathrm{~nm}$, the optimum for silica fibres. Both 1300 and $1550 \mathrm{~nm}$ lasers are based on quaternary materials such as GalnAsP, which gives a wide choice of operating wavelength in this region, and allows an important extra degree of freedom in device design while maintaining minimum stress and good reliability with the required optical waveguide and bandgap parameters.

An IRW (inverted rib waveguide) laser structure with a predicted failure rate of less than 1 in 60 over 25 years of operation has been announced. This is for use in single mode submarine systems.

The devices described so far use the accurately parallel cleaved facets as a pair of Fabry-Perot mirrors to give the necessary optical reinforcement for laser action. This is inherently capable of supporting several modes leach corresponding to a different integral number of wavelengths within the length of the structure), thus giving several optical lines spaced by the order of $1 \mathrm{~nm}$. The resulting 4-6 $\mathrm{nm}$ linewidth is undesirable for long-haul optical systems, particularly at $1500-1600 \mathrm{~nm}$ wavelengths where chromatic dispersion of silica fibre is high. For the full exploitation of fibres, a more ambitious goal has been set of a single, very narrow and stable spectral line. This enables signals to be transmitted at data rates of more than $1 \mathrm{Gbit} / \mathrm{s}$ over distances of more than $100 \mathrm{~km}$. To achieve this single line operation, a distributed feedback replaces the discrete optical feedback of the cleaved surface. An optical grating is incorporated into the device, either at the device surfaces, or (for better reliability) buried within it. This can take the form shown in Fig. 4. The effect of the grating is analogous to that of a multilayer coating, which can be designed for many small reflections to add in phase at a sharply defined wavelength. The laser wavelength is then defined precisely by the pitch of the internal grating and its refractive index. In addition to the very narrow linewidth achieved, DFB lasers are much easier to incorporate into planar integrated opti-

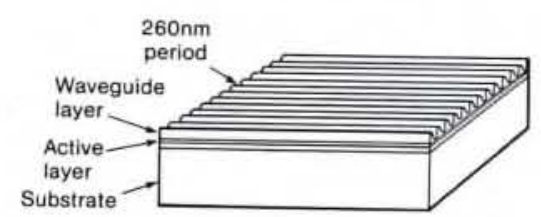

1. Grow planar double heterostructure and etch DFB grating

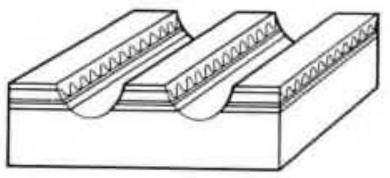

3. Etch double channel mesa

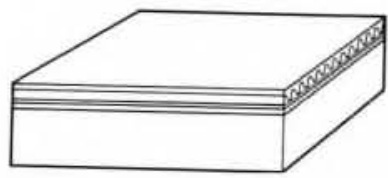

2. Overgrowth of grating

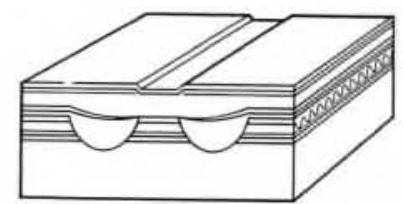

4. Buried heterostructure infill growth and apply stripe contact

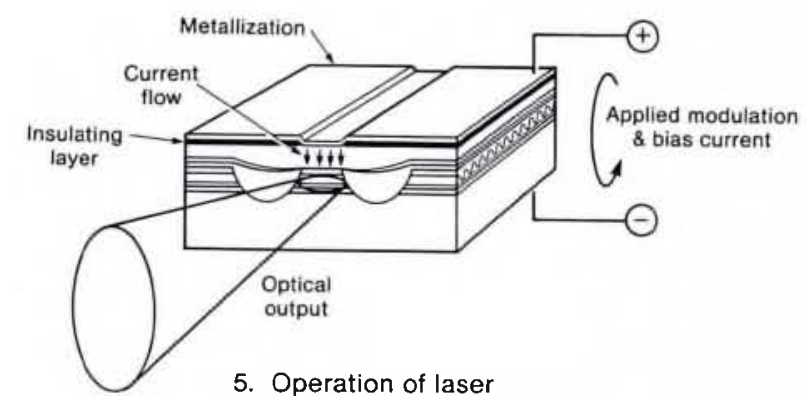

5. Operation of laser

Fig. 4 - Fabrication steps and operation of a distributed feedback laser. cal circuits because they do not require external mechanically cleaved facets. This opens up a wide range of possibilities for the future.

\section{Passive Optical Components}

Passive optical components such as device-to-fibre couplers, connectors and splitters/combiners are common requirements of many systems, and there are other more specialist requirements such as isolators (non-reciprocal one-way devices) or optical switches. STL has developed a wide range of components and techniques such as splicing suitable for field installation and maintenance of practical optical systems.

For multimode systems suitable for military field use, a typical optical connector is capable of withstanding the rigours of operational handling such as being dropped unmated into mud! The principle is indicated in Fig. 5. The fibre end is rigidly fixed at the focus of the lens and the fibre/lens units must then be aligned in a demountable connector.
The illustration shows how considerable lateral and longitudinal displacement between the units can be tolerated, and it is also a major advantage that dirt particles (large enough to black out a fibre/ fibre-end coupling) have very little effect. By providing a plane window, the device can be designed for easy cleaning. The trade-off is that angular alignment becomes much more critical than for a butt joint (as shown in the figure), perhaps $1^{\circ}$ or less, but this alignment accuracy is readily achievable. Such a connector, based on the use of specially designed thick plano-convex micro lenses ${ }^{3}$ ) is being used on rugged cable assemblies.

High strength splices also enable lengths of fibre to be joined to make long cable lengths, without the need to change athe cable dimensions in the region of the splices. Splices are produced using an electric arc (Fig. 6) as the heat source. In this equipment fibres are first aligned visually, using two perpendicular microscopic views, with fibre

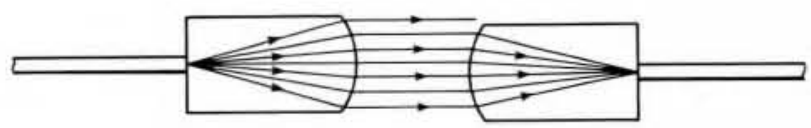

Relaxed tolerance to radial and longitudinal displacements

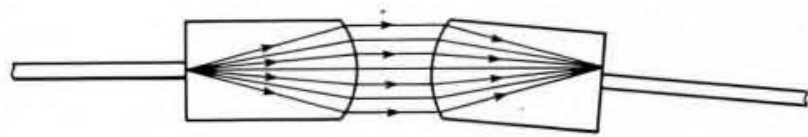

Tighter tolerance to angular misalignment

Fig. 5 - Principle of the expanded beam connector. 


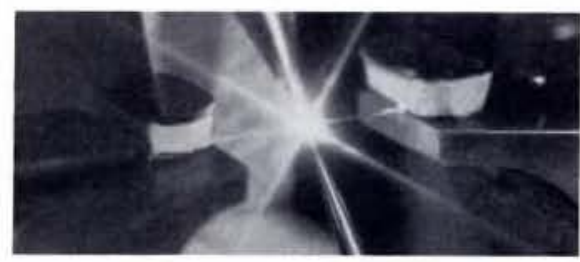

Fig. 6 - Arc fusion splicing of optical fibres.

ends in close proximity. The fibres are then automatically drawn apart, the pair of tungsten points brought in place and a controlled arc struck. The fibres are now automatically moved towards each other and into the hot zone where they soften and fuse on contact. For a good splice, microscopic inspection shows an undetectable joint on the outer surface. With STL-developed semi-automatic equipment, splice losses of less than 0.1 $\mathrm{dB}$ and mean strengths of $2-3 \%$ strain are achieved.

A range of fibre-based optical components is being developed in which optical functions, such as increased launch efficiency into a fibre from a laser, power splitting/combining, wavelength and polarisation multiplexing, power attenuation, switching, and amplitude and phase modulation, are performed within a length of optical fibre. The launch efficiency from a laser into a single-mode fibre has been increased by a factor of three over a butt launch by using a tapered spherical lens formed at the end of a single-mode fibre. To match the optical output pattern from a laser, generally more complex ellipsoidal lenses can be formed, integral with the fibre end.

Extensive development of biconical fused tapered coupler techniques, particularly for single-mode fibres, has led to the fabrication of power splitting couplers with excess losses of about $0.1 \mathrm{~dB}$, and wavelength multiplexers with channel separations ranging from $200 \mathrm{~nm}$ $(1300 / 1500 \mathrm{~nm}$ window multiplexing) to less than $10 \mathrm{~nm}$ in a single fibre transmission window. In addition, polarisation splitters based on these techniques have also been produced. Fig. 7 illustrates the principle of a $3 \mathrm{~dB}$ splitter, in which two single-mode fibres are laid side by side, fused and tapered over a short, controlled length. The power- and refractive-index profiles are illustrated at a few points along the length, from which it can be seen that power is shared between the two fibres. For a longer fused region the power would be periodically transferred between cores (as in a directional coupler, analogous to coupled pendulums). The periodic transfer of power depends upon wavelength, and this effect forms the basis of such useful devices as wavelength multiplexers. The illustration indicates the simple case of $50 \%$ of the launched power reaching each arm, and in practice this figure would be $49 \%$ (for a typical excess loss of $0.1 \mathrm{~dB}$ ).

\section{System Development}

From the early days of system work in the late 1960 s, STL has maintained a group on system-oriented component evaluation, which ensured that compo nent and fibre development progressed in close sympathy with system requirements. From the start, laser design was aimed at efficient coupling into fibres, a capability of fast modulation, and good spectral properties. Similarly, the first

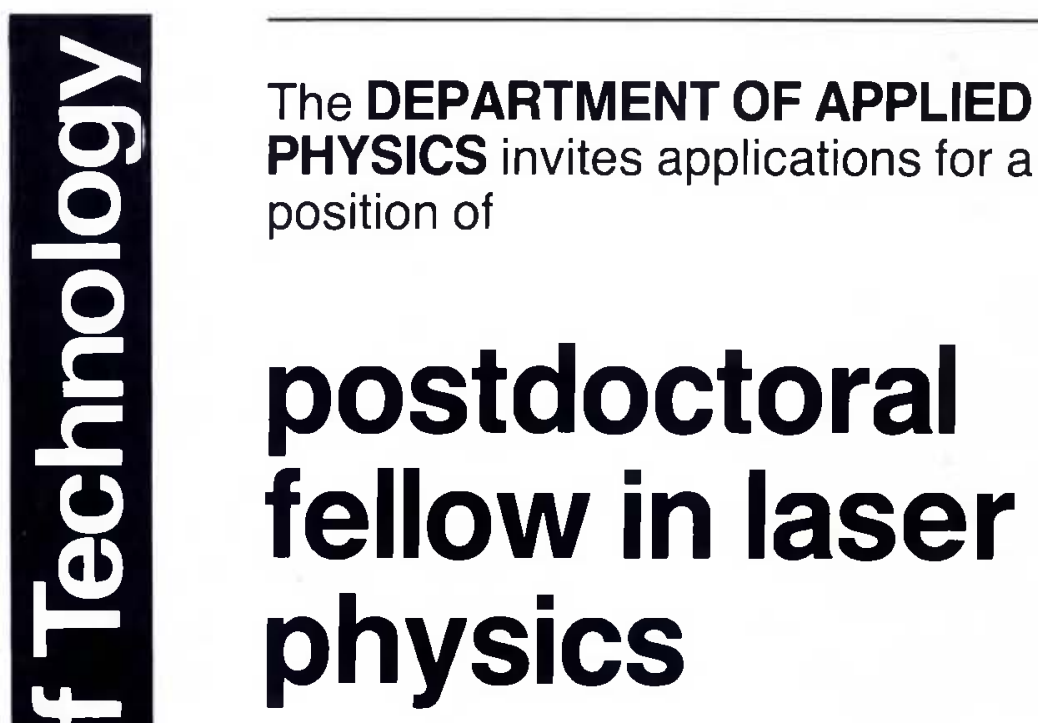

The appointed candidate will belong to the staff of the Quantum Electronics Group, where at present research is being conducted in the fields of pulsed multi atmosphere $\mathrm{CO}_{2}$ lasers, $\mathrm{AM}$ and injection mode locking, continuous and pulsed $\mathrm{CO}$ lasers, and E-beam and discharge excimer lasers.

The postdoctoral appointment is for one year and may be renewable for a second year; it will be sponsored by the Dutch Research Foundation (FOM). A competitive salary and 24 days annual vacation will be provided.

Please send your resume and references, quoting no. 2493/85/13144, to: Prof. Dr. Ir. W.J. Witteman, Dept. of Applied Physics, Twente University of Technology, P.O. Box 217, 7500 AE Enschede, The Netherlands. 


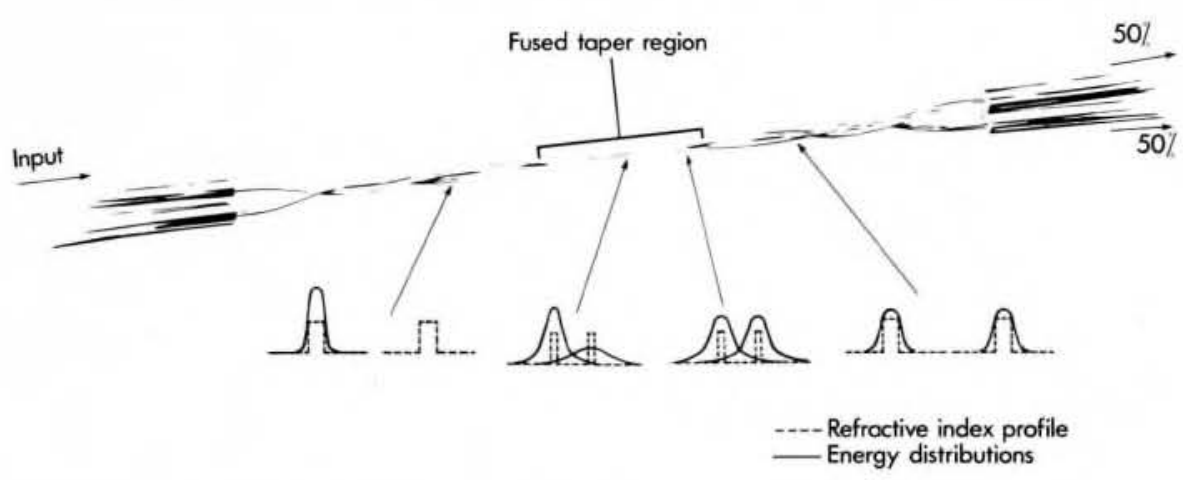

Fig. 7 - Fused tapered fibre coupler (3 dB type).

fibre dispersion measurement was developed by this group, and it continues to be true that systems and components need to be developed in close harmony.

Fundamental thinking is applied to understanding the physics of the system more exactly. A conventional system can be largely understood by treating the optical power as the time-varying signal with launched power drive current, received power $\sim$ launched power, and detector current $\sim$ received power. However, this simple picture is often inadequate as the signal actually consists of many electro-magnetic modes over a narrow range of frequencies and at various phases which give rise due to interference effects. A full electromagnetic analysis of the system including the degree of coherence of the optical source results in design criteria necessary to avoid random effects, such as modal noise.

In addition to predicting and controlling system limitations in this way, the fundamental approach also leads to opportunities for future advancement. With a single frequency optical transmitter one can adopt many of the radio techniques, using an optical frequency in place of RF. An example of such coherent techniques is the heterodyne receiver. Instead of direct detection of optical power, the received optical signal can be combined with an optical local oscillator (with a precisely controlled offset from the transmitter frequency) and a beat difference frequency carrying the signal information is then detected. The detected current will be proportional to $\sqrt{ } P_{0} P_{\mathrm{s}}$ rather than to $P_{\mathrm{s}}$ as would be the case for direct detection, where $P_{\mathrm{s}}$ and $P_{\mathrm{s}}$ are optical power levels from the local oscillator and received signal respectively. $\mathrm{By}$ providing sufficiently large local oscillator power, $\sqrt{ } P_{\mathrm{o}} P_{\mathrm{s}}$ can be made to dominate over receiver noise levels, resulting in significantly improved sensitivity for the heterodyne receiver. The first commercial application from these studies at STL was the coherent reflectometer, using the radar-like pulse echo techni- que for determining quality along a fibre and at splices, the highly sensitive heterodyne receiver giving an impressive range capability. The construction of a coherent system ${ }^{4}$ ) for point-to-point transmission, which is more difficult because the transmitter and LO sources are no longer co-located, has demonstrated that considerable improvements in receiver sensitivity and system capacity are feasible.

\section{Optical Sensors and Gyro}

One of the simplest concepts of optical sensors is where two fibres are aligned, and any minute relative movement of the fibres or change in the medium between them detected as a change of optical power. There are many practical inventions which depend on analysing the pattern of changes in optical intensity as a result of environmental changes, one successful example being to monitor oil-pollution levels within sea water. There is even more scope using the coherent optics approach, for example incorporating interference effects which allow extremely small changes within the fibre to be detected.

The fibre optical gyro achieves the purpose of keeping track of orientation by feeding a computer with continuous measurements of rate of rotation (angular velocity in three dimensions). This is in contrast to the navigation gyro in its pends on fixed reference directions given by the physical orientation of the gimbal-mounted gyroscope which are unaffected by twisting and turning.

The fibre optical gyro depends on the Sagnac effect, which deals with relativistic effects when a beam of light travels on a closed circuit (e.g. via mirrors). When the complete set-up of light source and mirrors rotates "with respect to the fixed stars" the round-trip time will depend on the rate of rotation. If the path takes the form of glass fibre, the effect is modified by "Fresnel-Fizeau drag", but the Sagnac phase remains. traditional mechanical form, which de-
To make such a tiny effect even remotely detectable, many turns of fibre are used, and light is sent both ways at once in order to detect the difference in traverse time between clockwise and anticlockwise paths. Extreme accuracies are needed, with typically $0.01 \% \mathrm{hr}$ required for a navigation gyro. To detect these path differences, optical phase has to be utilised, with the requirement of measuring extremely small fractions of an optical cycle.

\section{Conclusions}

Fibre optics technology has progressed from a physicist's dream to practical commercial systems which have been installed throughout the world, and continue to expand. This does not mean that the scientist's job is complete; there is a need for work on new materials for components and fibre, a deeper insight into the physics of failure mechanisms if we are to be sure of their reliability, a better understanding of advanced component manufacturing processes, and a knowledge of the fundamental mechanisms in the working optical systems. To this must be added the imagination to see more applications of the technology, many of which go far beyond the original concept of optical communication.

There is an exciting future in the research into longer wavelength materials, new coatings for fibres, new applications and advanced components, with the establishment of MOCVD, MBE and E-Beam epitaxy and lithographic techniques. One can forsee the evolution of public and private networks, multi-rate, multi-service broadband switching, with prospects of portable image phones, flat-screen pocket computers and optical signal processing being but some of the possibilities for the future.

\section{REFERENCES}

1. Kao K.C. and Hockham G.A., "Dielectricfibre surface waveguides for optical frequencies", IEE Proceedings 113 (July 1966) 7, 1151-1158.

2. Renner D., Greene P.D., Collar A.J. and Moule D.J., "Very low threshold current GalnAsP lasers", IEE Colloquium, Towards Semiconductor Integrated Optoelectronics, 25 March 1985, 3/1-6.

3. Cooper P.R., Leach J.S., Harding A.B. and Matthews M.A., "A fibre optic connector suitable for use in a rugged environment", Optics and Laser Technology, 14 (April 1982) 2, 87-91.

4. Davis A.W. and Wright S., "A wideband homodyne receiver using phase diversity", presented at the 11th European Conference on Optical Communication, Venice, 1-4 October 1985. 